\title{
Determinants of the essential one-carbon metabolism metabolites, homocysteine, S-adenosylmethionine, S-adenosylhomocysteine and folate, in cerebrospinal fluid
}

\author{
Desirée E.C. Smith ${ }^{1}$, Yvo M. Smulders ${ }^{2,3}$, Henk J. \\ Blom $^{1,3}$, Julius Popp ${ }^{4,5}$, Frank Jessen ${ }^{4}$, Alexander \\ Semmler ${ }^{6}$, Melinda Farkas ${ }^{6}$ and Michael Linnebank ${ }^{6, *}$ \\ ${ }^{1}$ Department of Clinical Chemistry, Metabolic Laboratory, \\ VU University Medical Center, Amsterdam, \\ The Netherlands \\ ${ }^{2}$ Department of Internal Medicine, VU University Medical \\ Center, Amsterdam, The Netherlands \\ ${ }^{3}$ Institute for Cardiovascular Research ICaR-VU, VU \\ University Medical Center, Amsterdam, The Netherlands \\ ${ }^{4}$ Department of Psychiatry, University Hospital Bonn, Bonn, \\ Germany \\ ${ }^{5}$ Department of Psychiatry, University Hospital of Lausanne, \\ Lausanne, Switzerland \\ ${ }^{6}$ Department of Neurology, University Hospital Zurich, \\ Zurich, Switzerland
}

\begin{abstract}
Background: Disturbances in the levels of one-carbon (1C) metabolism metabolites have been associated with a wide variety of neuropsychiatric diseases. Cerebrospinal fluid (CSF) levels of homocysteine (Hcy) and the other 1C metabolites, nor their interrelatedness and putative determinants, have been studied extensively in a healthy population.

Methods: Plasma and CSF samples from 100 individuals free from neuropsychiatric diseases were analyzed (55 male, 45 female; age $50 \pm 17$ years). In blood, we measured plasma Hcy, serum folate and serum vitamin B12. In CSF, we measured total Hcy, S-adenosylmethionine (SAM), S-adenosylhomocysteine (SAH) and 5-methyltetrahydrofolate (5-methylTHF). Highly selective analytical methods like liquid chromatography combined with either mass spectrometry or fluorescence detection were used.

Results: CSF Hcy was inversely correlated with CSF 5-methylTHF and positively with plasma Hcy, independent of serum folate status. CSF SAH correlated with age, lower CSF 5-methylTHF and higher CSF Hcy. CSF 5-methylTHF showed independent negative correlations with age and

*Corresponding author: PD Dr. Michael Linnebank, Department of Neurology, University Hospital Zurich, Frauenklinikstr. 26, 8091

Zurich, Switzerland

Phone: +41 44 2551111, Fax: +41 442554507 ,

E-mail: michael.linnebank@usz.ch

Received August 18, 2011; accepted February 27, 2012;

previously published online March 24, 2012
\end{abstract}

positive correlations with serum folate. CSF SAM did not correlate with any of the $1 \mathrm{C}$ metabolites.

Conclusions: Aging is characterized by a reduction in CSF 5-methylTHF levels and increased CSF levels of the potentially neurotoxic transmethylation inhibitor SAH. CSF 5-methylTHF, which is itself determined in part by systemic folate status, is a powerful independent determinant of CSF levels of Hcy and SAH.

Keywords: cerebrospinal fluid; folate; homocysteine; S-adenosylhomocysteine; S-adenosylmethionine.

\section{Introduction}

One-carbon (1C) metabolism encompasses a series of biochemical reactions that involves the transfer of $1 \mathrm{C}$ moieties (Figure 1). One of its main purposes is to provide methyl groups for virtually all transmethylation reactions. One carbon metabolism has been the focus of extensive research, particularly since increased plasma levels of one of its intermediates, homocysteine (Hcy), were linked to numerous diseases, such as cardiovascular disease (3) and neurological disease $(4,5)$.

Studies with small sample sizes have also shown correlations of these neurological diseases with Hcy in cerebrospinal fluid (CSF) $(6,7)$. To date, however, several uncertainties surround the association between increased plasma levels of Hcy and neuropsychiatric disease. Firstly, it is unclear whether plasma or CSF concentrations of these constituents are primarily of interest. In addition, it remains unclear whether Hcy itself or one of the other constituents of 1C metabolism exerts detrimental effects on the neural system. Potential candidates in this context include S-adenosylmethionine (SAM), S-adenosylhomocysteine (SAH) and B-vitamin status.

SAM is the methyl donor in many essential methylation reactions, such as DNA methylation, synthesis and inactivation of neurotransmitters, and myelin synthesis. SAH has opposite effects, in that it acts as a powerful inhibitor of most transmethylation reactions (8). The SAM/SAH ratio has been used as a reflection of methylation potential in, amongst others, the central nervous system (CNS) (9). The potential relevance of SAM is supported by observations that low SAM levels in CSF are associated with depression and dementia (10). The effect of SAH on neuropsychiatric disturbances is less well studied. However, the CSF levels of SAH are associated with CSF levels of hyperphosphorylated tau, a marker for Alzheimer pathology (11). 


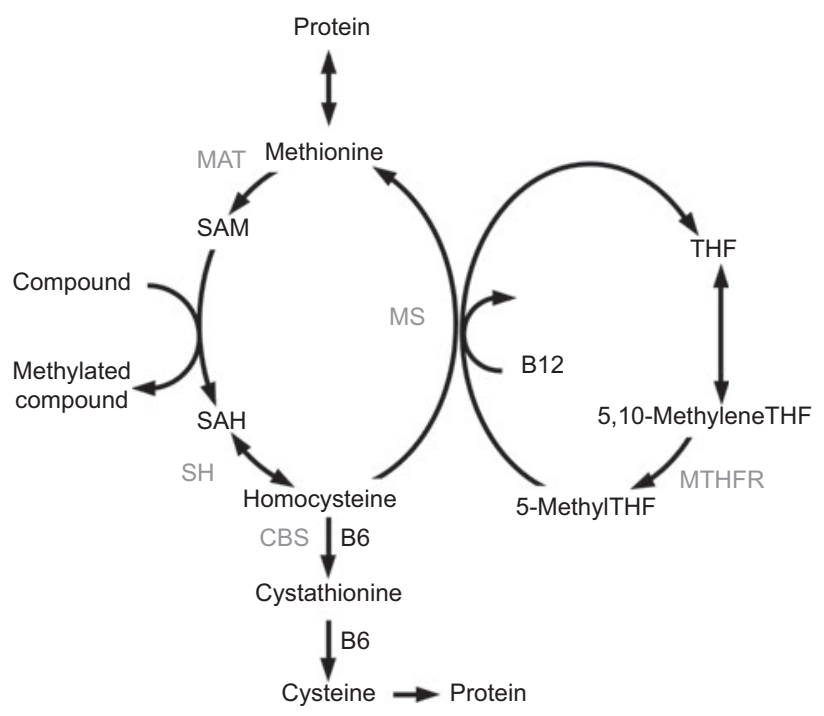

Figure 1 Overview of the 1 carbon metabolic pathway in humans. Homocysteine (Hcy) can follow two pathways.

It could be remethylated to methionine and subsequently converted to the universal methyl donor S-adenosylmethionine (SAM). Disturbed remethylation influences methylation reactions, such as the methylation of neurotransmitters or DNA. Alternatively, Hcy can be irreversibly converted to cystathionine and further to cysteine. However, whether this transsulfuration pathway is active in the brain, is still debated (1). Elevated Hcy levels could also influence SAH levels by the equilibrium reaction. High SAH is an inhibitor of a wide variety of methyltransferases (2). MS, methionine synthase; MTHFR, methylene tetrahydrofolate reductase; CBS, cystathionine $\beta$-synthase; MAT, methionine adenosyl transferase; $\mathrm{SH}$, SAH hydrolase.

One-carbon metabolism is critically dependent on the B-vitamin status (B2, B6, B12 and, predominantly, folate) (12). Deficiencies of B-vitamins are commonly seen in the elderly population $(13,14)$. As the remethylation pathway via betaine is not expressed in the brain, the only option for remethylation of Hcy, and thus regeneration of methionine, is via 5-methyltetrahydrofolate (5-methylTHF, Figure 1). Therefore, low 5-methylTHF levels could not only increase Hcy levels but could also potentially diminish the methylation capacity of the brain (15). Additionally, folates are involved in DNA synthesis; also low folate levels could potentially impair DNA stability and structure, for example by the misincorporation of uracil in DNA (16). The literature on folate status as an independent risk factor for neuropsychiatric diseases indicates that low levels of 5-methylTHF in CSF are found in patients suffering from a wide spectrum of neuropsychiatric diseases, such as Alzheimer, depression and autism (17-20). Additionally, the absence of 5-methylTHF in CSF in rare inborn errors of metabolism causes progressive neurological decline (21).

In short, concentrations in the CNS of Hcy, but also of other 1C metabolites, in particular SAM, SAH and 5-methylTHF, may be of relevance for the development of neuropsychiatric diseases. A better understanding of the determinants of these intermediates is important. This paper focuses on identification of determinants of Hcy, SAM, SAH and 5-methylTHF in CSF of individuals unaffected by neuropsychiatric disease. Additionally, the reference values in this paper will offer an indication for the sample size estimation of future studies.

\section{Materials and methods}

\section{Subjects}

Ninety-eight subjects, who were subjected to a lumbar puncture (for different indications, such as exclusion of CNS inflammation, exclusion of aneurysmal subarachnoid hemorrhage or exclusion of meningitis, all negative) at the Department of Neurology (University of Bonn), were enrolled in this study. Except for the symptoms they presented with, majorly acute, but not chronic cepahlgia, none of the enrolled subjects showed evidence or history of cognitive impairment or any other symptoms of possible neurological or psychiatric disease; which were exclusion criteria. Additional exclusion criteria for this study were: chronic or unstable medical illness (e.g., symptomatic cardiac disease, renal or hepatic dysfunction, insulindependent diabetes mellitus, untreated thyroidal dysfunction), excessive alcohol intake, vitamin supplementation, disturbance of the blood brain-barrier (defined as CSF whole protein content $>500 \mathrm{mg}$ / $\mathrm{dL}$ ), or inflammation of the central nervous system (i.e., more than 5 leucocytes $/ \mathrm{mm}^{3}$ or intrathecal immunglobulin production) or an abnormal result in the Mini Mental State Examination (MMSE), which was done for all subjects (22). The study was approved by the Local Ethics Committee, written informed consent was obtained from all study participants.

\section{Sample collection}

Diagnostic lumbar punctures were performed at the Department of Neurology, University of Bonn. A standardized technique with a 20G "atraumatical" spinal needle and a sitting or lying position for the patient was applied. Approximately $10-14 \mathrm{~mL}$ of CSF was removed for analysis. Depending on the indication of the lumbar puncture, the first fractions were used for diagnostic purposes, the third or fourth for the current study. CSF samples were frozen immediately on dry ice.

Fasting venous blood was collected in lithium heparin vacutainers and immediately placed on ice. Plasma was isolated by centrifugation for $10 \mathrm{~min}$ at $2000 \mathrm{~g}$ at $4^{\circ} \mathrm{C}$. Light protected sera were used for the folate and vitamin B12 measurements; these were centrifuged for $10 \mathrm{~min}$ at $3300 \mathrm{~g}$ at room temperature. All samples were stored at $-80^{\circ} \mathrm{C}$ until analysis.

\section{Methods}

Plasma total Hcy concentrations were determined by means of fully automated particle-enhanced immunonephelometry with a BN II System (Siemens Healthcare Diagnostics $\mathrm{GmbH}$, Eschborn, Germany). The intraassay and interassay coefficient of variation (CV) of the homocysteine assay were $3.4 \%$ and $5.6 \%$, respectively.

Serum vitamin B12 and folate concentrations were determined by means of a competitive chemiluminescent immunoassay with an Access ${ }^{\mathrm{TM}}$ Immunoassay System (Beckman Coulter, Krefeld, Germany). The intraassay and interassay CVs of the vitamin B12 assay were $3.8 \%$ and $6.3 \%$, 
respectively. The intraassay and interassay CVs of the folate assay were $2.8 \%$ and $4.8 \%$, respectively.

CSF SAM and SAH concentrations were determined by liquid chromatography-tandem mass spectrometry (LC-MS/ MS) (API3000, Applied Biosystems, Foster City, CA, USA). The intraassay and interassay CVs for SAM were $6.8 \%$ and $4.2 \%$, respectively. The intraassay and interassay CVs for SAH were $6.9 \%$ and $5.5 \%$, respectively (23).

CSF folate vitamers were determined by LC-MS/MS (API3000, Applied Biosystems). Intraassay and interassay CVs for 5-methylTHF were $1.2 \%$ and $2.8 \%$, respectively. Intraassay and interassay $\mathrm{CVs}$ for non-methylTHF as a group were $1.6 \%$ and $1.5 \%$, respectively (24).

CSF total Hcy concentrations were measured by HPLC using fluorescence detection (Waters, Milford, MA, USA). An adaptation of a previously published method was used (25). In order to be able to measure the nanomolar homocysteine concentration in $100 \mu \mathrm{L}$ of CSF accurately, the reaction volume was minimized and a flushing gradient (30\% acetonitrile) was added after each injection to create a more stable HPLC baseline. The intraassay and interassay CVs were $2.2 \%$ and $3.6 \%$, respectively.

\section{Statistical analysis}

All statistical analyses were performed using SPSS 17.0 for Windows. Most of the measured parameters showed positively skewed distributions (plasma Hcy, CSF Hcy, serum folate, serum B12, CSF SAH, CSF SAM/SAH), so the data were $\log$ transformed prior to regression analysis. As outlined in the Introduction, the main dependent variables of interest in CSF were Hcy, SAM, SAH, SAM/SAH and 5-methylTHF. We performed separate analyses to identify determinants of these CSF constituents.

In order to reduce the risk of finding associations by chance, a priori hypotheses regarding plausible determinants of the dependent variables of interest were formulated using available knowledge of 1C metabolism and epidemiology. For CSF Hcy, we first assessed traditional determinants known to influence plasma Hcy, namely age and B-vitamin status (in both plasma and CSF). In order to determine whether serum folate status would determine CSF Hcy via its correlation with CSF folate status, serum folate and CSF 5-methylTHF were analyzed in a multiple regression model. In addition, we analyzed the correlation between plasma vs. CSF Hcy, and adjusted associations between serum folate status and CSF Hcy for plasma Hcy. All significant determinants of CSF Hcy were finally entered into a single multiple regression model.

For CSF SAM, SAH and their ratio, the following potential determinants were analyzed: age, serum B vitamin status, plasma and CSF Hcy and CSF 5-methylTHF. Positive correlations were analyzed for independence, again with multiple regression analysis

Finally, for CSF 5-methylTHF, we assessed whether its concentration independently correlated with age and/or serum folate status and whether the CSF 5-methylTHF/serum folate ratio correlated with age.
Table 1 Study population characteristics.

\begin{tabular}{lc}
\hline Age, years & $50 \pm 17$ \\
Gender, male/female, $\mathrm{n}$ & $55 / 45$ \\
Plasma homocysteine, $\mu \mathrm{M}$ & $11(4-34)$ \\
Serum total folate, $\mathrm{nmol} / \mathrm{L}$ & $11(2-39)$ \\
Serum vitamin $\mathrm{B} 12, \mathrm{pg} / \mathrm{mL}$ & $325 \pm 126$ \\
CSF homocysteine, $\mathrm{nmol} / \mathrm{L}$ & $61(13-303)$ \\
$\mathrm{CSF}$ 5-methylTHF, nmol/L & $42 \pm 14$ \\
CSF non-methylTHF, nmol/L & $0(0-12)$ \\
Non-methylTHF, \% & $0(0-31)$ \\
CSF SAM, nmol/L & $205 \pm 41$ \\
CSF SAH, nmol/L & $22(11-49)$ \\
CSF SAM/SAH & $9(3-20)$ \\
\hline
\end{tabular}

Data are presented as mean (SD) or, in case of skewed distributions, as median (range).

As most of the measured parameters were log transformed, interpretation of the effect size of a given determinant on the investigated $1 \mathrm{C}$ metabolite is difficult. In order to display the effect sizes more clearly, we divided the non-logarithmic transformed levels of the investigated metabolites into tertiles, repeated the multivariate analyses and plotted the increase in the dependent variable in tertiles, with the first tertile as reference.

\section{Results}

Characteristics of included individuals are listed in Table 1. CSF values for Hcy, folate, SAM, and SAH compared well with a previous reported study (9). Some individuals showed hyperhomocysteinemia in plasma, which coincided with low serum folate levels. Subjects were not excluded for this reason. With regard to the comparison of $1 \mathrm{C}$ metabolites levels in CSF to plasma, CSF Hcy levels were a 100-fold lower than the plasma levels. Remarkably, CSF folate levels were substantially higher than plasma levels. SAM and SAH levels in CSF were comparable to plasma levels, previously determined

Table 2 Univariate and multivariate regression models of determinants of CSF Hcy.

\begin{tabular}{|c|c|c|c|c|}
\hline $\begin{array}{l}\text { Regression } \\
\text { type }\end{array}$ & Dependent & Independent & $\beta$ & p-Value \\
\hline Univariate & CSF Hcy ${ }^{c}$ & Age & 0.181 & 0.075 \\
\hline Univariate & CSF Hcy ${ }^{c}$ & CSF 5-methylTHF & -0.495 & $0.000^{\mathrm{b}}$ \\
\hline Univariate & CSF Hcy ${ }^{c}$ & Plasma Hcyc & 0.331 & $0.001^{\mathrm{b}}$ \\
\hline Univariate & $\mathrm{CSF}^{\mathrm{Hcy}}{ }^{\mathrm{c}}$ & Serum folate $\mathrm{c}^{\mathrm{c}}$ & -0.244 & $0.019^{\mathrm{a}}$ \\
\hline Univariate & CSF Hcy ${ }^{c}$ & Serum B12c & -0.176 & 0.094 \\
\hline Univariate & CSF Hcy ${ }^{c}$ & $\mathrm{CSF}_{\mathrm{SAH}}^{\mathrm{c}}$ & 0.380 & $0.000^{\mathrm{b}}$ \\
\hline \multirow[t]{2}{*}{ Multivariate 1} & $\mathrm{CSF}_{\mathrm{Hcy}}{ }^{\mathrm{c}}$ & CSF 5-methylTHF & -0.499 & $0.000^{\mathrm{b}}$ \\
\hline & & Serum folate ${ }^{c}$ & 0.008 & 0.943 \\
\hline \multirow[t]{2}{*}{ Multivariate 2} & $\mathrm{CSF} \mathrm{Hcy}^{\mathrm{c}}$ & Plasma Hcy & 0.279 & $0.016^{\mathrm{a}}$ \\
\hline & & Serum folate ${ }^{c}$ & -0.109 & 0.339 \\
\hline \multirow[t]{3}{*}{ Multivariate 3} & CSF Hcy ${ }^{c}$ & CSF 5-methylTHF & -0.338 & $0.001^{\mathrm{b}}$ \\
\hline & & Plasma Hcy ${ }^{c}$ & 0.204 & $0.027^{\mathrm{a}}$ \\
\hline & & CSF SAH ${ }^{c}$ & 0.265 & $0.004^{\mathrm{b}}$ \\
\hline
\end{tabular}

${ }^{a}$ Correlation is significant at the 0.05 level (2-tailed), ${ }^{\mathrm{b}}$ correlation is significant at the 0.01 level (2-tailed), ${ }^{c} \log$ transformed data. 
using similar analytical methods (26). Of note, folate existed in CSF predominantly in the 5-methylTHF form, as was previously described for plasma (27). With regard to gender, no significant correlations with any of the parameters were found (data not shown).

\section{Determinants of CSF Hcy}

For the potential determinants of CSF Hcy listed in the Methods section, the univariate correlations were determined. The results are listed in Table 2. CSF Hcy levels were positively correlated with plasma Hcy and CSF SAH, then negatively correlated with serum folate and CSF folate. Age, a traditional determinant of plasma Hcy, just failed to reach significance for CSF Hcy $(\mathrm{p}=0.075)$.
Multiple regression analysis was used to determine interdependence of the univariate correlations. As is evident from both multivariate regression model 1 and 2 in Table 2, serum folate is not an independent determinant of CSF Hcy. Rather, its correlation with CSF Hcy is predominantly explained by its effect on CSF 5-methylTHF and plasma Hcy.

As explained in Figure 1, the metabolic fate of Hcy is determined by several pathways, implying that its concentration could be independently correlated to parameters related to these pathways. Regression model 3 indeed identified CSF 5-methylTHF (remethylation), plasma Hcy (import/export across the blood-brain barrier) and CSF SAH (SAH hydrolase activity) as independent determinants of CSF Hcy levels. When age and vitamin B12 were forced into the multiple regression model, they were again not significantly related to
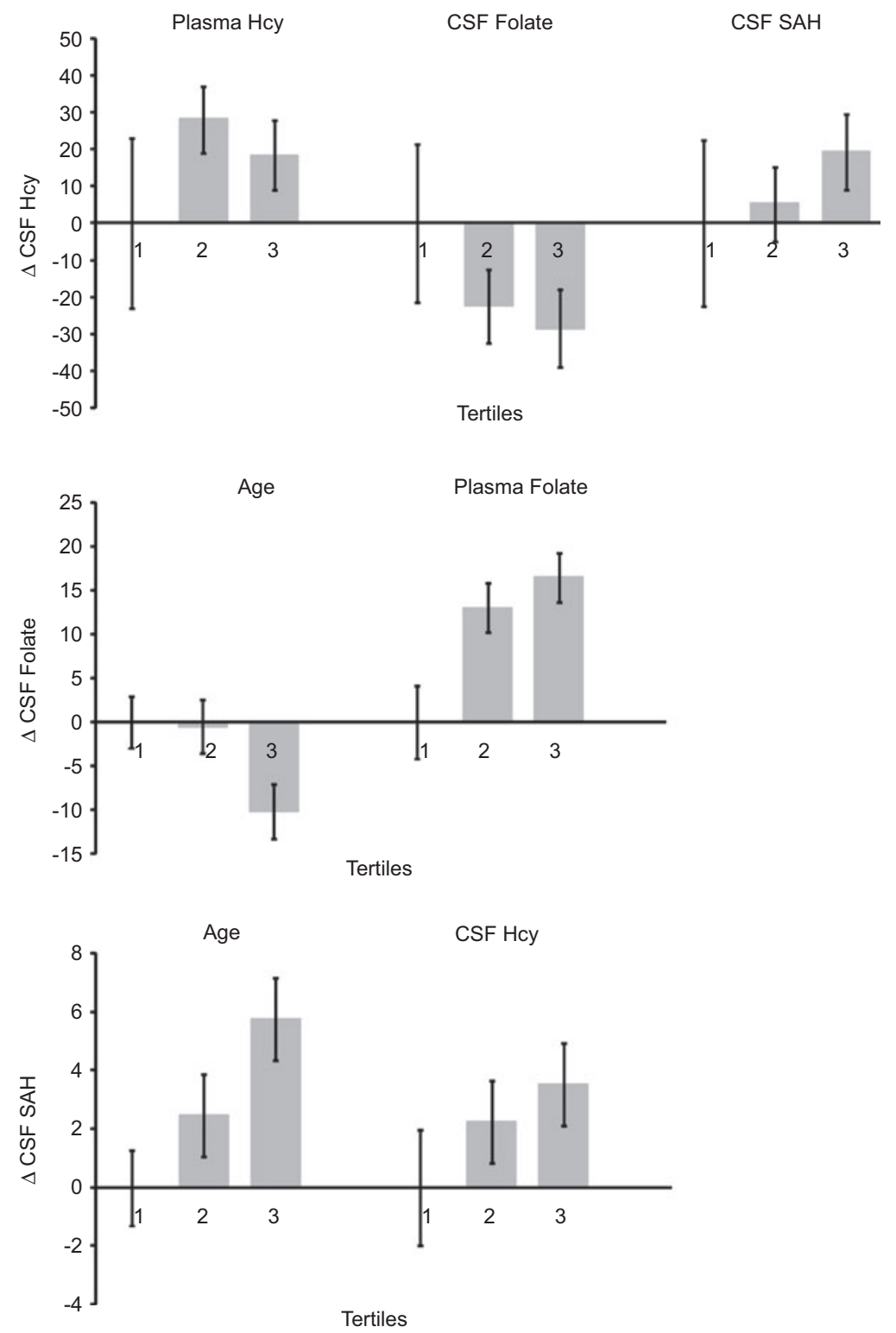

Figure 2 Change in CSF Hcy, CSF folate and CSF SAH, in respective tertiles (multivariate regression model). Effect sizes were adjusted for the variables as indicated in Table 2. 
CSF Hcy (data not shown). Figure 2 demonstrates the multiple adjusted impact of plasma Hcy, CSF 5-methylTHF and CSF SAH, on CSF Hcy.

\section{Determinants of CSF SAM, SAH and SAM/SAH}

As shown in Table 3, CSF SAM showed no significant correlation with any of the potential determinants studied. CSF SAH was independently positively correlated with age and CSF Hcy, and negatively correlated with CSF 5-methylTHF (Figure 2). The CSF SAM/SAH ratio was independently positively correlated with CSF 5-methylTHF and negatively correlated with age and CSF Hcy. None of the serum parameters (Hcy, folate and B12) showed any significant correlation with CSF SAM, SAH or the SAM/SAH ratio (data not shown).

\section{Determinants of CSF 5-methylTHF}

As shown in Table 4, CSF 5-methylTHF is negatively correlated with age and positively correlated with serum folate concentrations. A multivariate regression model for both age and serum folate was applied, showing that both parameters independently influence CSF 5-methylTHF (Figure 2). Additionally, the ratio CSF 5-methylTHF/serum folate was negatively correlated with age $\left(R^{2}=0.076, \beta=-0.276, \mathrm{p}=0.008\right)$.

\section{Discussion}

These are the salient findings of our study in healthy individuals. Firstly, older age and lower serum folate status are independently associated with lower CSF 5-methylTHF availability. This may be unfavorable, as we also observed that a low CSF folate status adversely affects CSF Hcy and methylation potential, at least insofar as the latter is reflected by concentrations of SAH. Secondly, aging also appears to directly adversely affect methylation potential, independent of CSF folate status. Finally, CSF Hcy appears to be at least partly determined by plasma Hcy, suggesting a degree of blood-brain equilibration. In the highest tertile of CSF Hcy, the contribution of plasma Hcy is less, suggesting that higher CSF Hcy is predominantly related to intracerebral metabolism, rather than to equilibration with plasma Hcy. CSF Hcy is independently correlated to the concentration of the transmethylation inhibitor SAH, which may represent a mechanism of untoward effects of CSF Hcy.

The fate of Hcy in CSF will depend on many different factors (remethylation, export from brain tissue, transport across the blood-brain barrier, equilibrium with $\mathrm{SAH}$, etc). From the perspective of Hcy metabolism (Figure 1), the positive correlation of CSF Hcy with both plasma Hcy and CSF SAH, and the negative correlation with CSF 5methylTHF seem logical (9). However, given the strong degree of inter-relatedness of $1 \mathrm{C}$ metabolites, analyzing statistical independency of these associations, as we did in the current study, is crucial.

The methylation potential of cells is conceivably reflected by SAM and SAH (and their ratio), as SAM is a universal methyl donor and SAH is an inhibitor of most transmethylation reactions. However, in line with previously reported data, CSF SAM levels did not correlate with any of the other studied potential determinants (9). From a teleological perspective, this could mean that keeping CSF SAM levels stable is vitally important, which would be in keeping with its central role not only as a methyl donor, but also as a regulator of enzyme activity in both the remethylation and transsulfuration pathway. The fact that significantly lower amounts of SAM were measured in CSF of patients suffering from depression and dementia, may offer insight into how disturbances in $1 \mathrm{C}$ metabolism and this type of diseases are linked (10). It may be that intracellular concentrations in brain tissue obtained from specific regions will offer more insight into these matters,

Table 3 Univariate and multivariate regression models of determinants of CSF SAM, SAH and SAM/SAH.

\begin{tabular}{|c|c|c|c|c|}
\hline $\begin{array}{l}\text { Regression } \\
\text { type }\end{array}$ & Dependent & Independent & $\beta$ & $\mathrm{p}$-Value \\
\hline Univariate & CSF SAM & Age & 0.033 & 0.750 \\
\hline Univariate & CSF SAM & CSF 5-methylTHF & 0.093 & 0.365 \\
\hline Univariate & CSF SAM & CSF Hcy ${ }^{c}$ & 0.164 & 0.107 \\
\hline Univariate & $\mathrm{CSF} \mathrm{SAH}^{\mathrm{c}}$ & Age & 0.462 & $0.000^{\mathrm{b}}$ \\
\hline Univariate & $\mathrm{CSF} \mathrm{SAH}^{\mathrm{c}}$ & CSF 5-methylTHF & -0.321 & $0.001^{\mathrm{b}}$ \\
\hline Univariate & $\mathrm{CSF}_{\mathrm{SAH}}{ }^{\mathrm{c}}$ & CSF Hcy ${ }^{c}$ & 0.380 & $0.000^{\mathrm{b}}$ \\
\hline Univariate & CSF SAM/SAH ${ }^{c}$ & Age & -0.405 & $0.000^{\mathrm{b}}$ \\
\hline Univariate & $\mathrm{CSF}$ SAM/SAH ${ }^{\mathrm{c}}$ & CSF 5-methylTHF & 0.361 & $0.000^{\mathrm{b}}$ \\
\hline Univariate & CSF SAM/SAH ${ }^{\mathrm{c}}$ & CSF Hcy ${ }^{c}$ & -0.252 & $0.012^{\mathrm{a}}$ \\
\hline \multirow[t]{3}{*}{ Multivariate } & CSF SAH & Age & 0.391 & $0.000^{\mathrm{b}}$ \\
\hline & & CSF 5-methylTHF & -0.097 & 0.336 \\
\hline & & $\mathrm{CSF} \mathrm{Hcy}^{\mathrm{c}}$ & 0.261 & $0.010^{\mathrm{a}}$ \\
\hline \multirow[t]{3}{*}{ Multivariate } & CSF SAM/SAH & Age & -0.333 & $0.001^{\mathrm{b}}$ \\
\hline & & CSF 5-methylTHF & 0.246 & $0.022^{\mathrm{a}}$ \\
\hline & & CSF Hcy ${ }^{c}$ & -0.070 & 0.499 \\
\hline
\end{tabular}

${ }^{a}$ Correlation is significant at the 0.05 level (2-tailed), ${ }^{b}$ correlation is significant at the 0.01 level (2-tailed), ${ }^{c} \log$ transformed data. All serum (Hcy, folate and B12) correlations were not significant. 
Table 4 Univariate and multivariate regression models of determinants of CSF 5-methylTHF.

\begin{tabular}{lllrl}
\hline $\begin{array}{l}\text { Regression } \\
\text { type }\end{array}$ & Dependent & Independent & $\beta$ & p-Value \\
\hline Univariate & CSF 5-methylTHF & Age & -0.242 & $0.016^{\mathrm{a}}$ \\
Univariate $^{\text {Multivariate }}$ & CSF 5-methylTHF & Serum folate & 0.504 & $0.000^{\mathrm{b}}$ \\
& CSF 5-methylTHF & Age & -0.256 & $0.004^{\mathrm{b}}$ \\
& & Serum folate $^{\mathrm{c}}$ & 0.511 & $0.000^{\mathrm{b}}$ \\
\hline
\end{tabular}

${ }^{\mathrm{a}}$ Correlation is significant at the 0.05 level (2-tailed), ${ }^{\mathrm{b}}$ correlation is significant at the 0.01 level (2-tailed), ${ }^{c} \log$ transformed data.

as CSF SAM does not correlate well with intracellular SAM concentrations in brain $(28,29)$.

Levels of the methyltransferase inhibitor SAH were adversely associated with higher age and CSF Hcy (9). Age seems to be a strong determinant of the methylation potential in the brain. The underlying mechanism remains unclear.

In our study, CSF folate levels were approximately five times higher than serum folate levels. Another study (18) showed similar results, while a further one (9) observed higher folate serum levels than in our population and lower CSF levels. However, this last study did not use multivitamin intake as an exclusion criteria. This may account for the higher plasma values. Perhaps folate demand in the brain is higher than in other organs, but exactly how the brain maintains its high folate status is mechanistically unknown. The relation between increased age and diminished serum folate levels has been well documented (14). This correlation has been attributed to the decreased ability for folate uptake in the elderly population $(13,14)$. Our study also showed that age was an independent determinant of CSF folate levels, a fact that has not been described before to our knowledge.

As CSF folate levels have been shown not to follow serum folate levels outside the normal range $(\geq 45 \mathrm{nmol} / \mathrm{L})(9)$, an additional determinant of CSF folate is of importance. Choroid plexus epithelial cells express high levels of folate receptor $\alpha$, suggesting that a role for it in folate trafficking (30). Perhaps transport of folate over the blood-brain barrier is also a determinant of CSF folate and might be the rate limiting step. The observation that the $\mathrm{CSF} /$ serum folate ratio diminishes with age may suggest that this transport alters with age. Supplementation might increase the often reduced plasma folate levels of the elderly. However, whether this supplementation will lead to desired increase in CSF folate has yet to be determined. Additionally, this observation of hindered transport of folate across the blood-brain barrier could provide insight to the origin of the low CSF folate levels observed in cerebral folate deficiency, a treatable neurological abnormality with genetic, acquired or even unknown etiology (31). Whether deficient CSF folate reflects an intracellular folate deficiency in the brain is not well understood. Because of the strong observed correlation between CSF folate and CSF Hcy, CSF Hcy could be considered to be a more practical biomarker (compound stability) of cerebral folate deficiency.

Obtaining CSF samples from healthy volunteers is difficult. Even though individuals taking medication or suffering from diseases known to influence the 1C metabolism were omitted, a certain degree of bias cannot be excluded.

Having identified the determinants of $1 \mathrm{C}$ metabolites in CSF, it will be interesting to investigate whether the same determinants play a role in various neurodegenerative diseases and whether various intervention strategies can alter CSF concentrations of the relevant $1 \mathrm{C}$ metabolites, offering pathways to prevention and/or treatment.

In conclusion, CSF 5-methylTHF levels decrease while CSF SAH levels increase with age, which may contribute to CNS ageing and neurodegeneration. Higher serum folate concentrations were associated with higher CSF concentrations of 5 -methylTHF and with lower levels of the neurotoxic agents Hcy and SAH, arguing that folate deficiency may accelerate ageing and neurodegeneration.

\section{Conflict of interest statement}

Authors' conflict of interest disclosure: The authors stated that there are no conflicts of interest regarding the publication of this article.

Research funding: None declared.

Employment or leadership: None declared.

Honorarium: None declared.

\section{References}

1. Griffiths R, Tudball N. Observations on the fate of cystathionine in rat brain. Life Sci 1976;19:1217-24.

2. James SJ, Melnyk S, Pogribna M, Pogribny IP, Caudill MA. Elevation in S-adenosylhomocysteine and DNA hypomethylation: potential epigenetic mechanism for homocysteine-related pathology. J Nutr 2002;132:2361S-6S.

3. Homocysteine Studies Collaboration. Homocysteine and risk of ischemic heart disease and stroke: a meta-analysis. J Am Med Assoc 2002;288:2015-22.

4. Obeid R, Herrmann W. Mechanisms of homocysteine neurotoxicity in neurodegenerative diseases with special reference to dementia. FEBS Lett 2006;580:2994-3005.

5. Stanger O, Fowler B, Piertzik K, Huemer M, Haschke-Becher E, Semmler A, et al. Homocysteine, folate and vitamin B12 in neuropsychiatric diseases: review and treatment recommendations. Expert Rev Neurother 2009;9:1393-412.

6. Isobe C, Murata T, Sato C, Terayama Y. Increase of total homocysteine concentration in cerebrospinal fluid in patients with Alzheimer's disease and Parkinson's disease. Life Sci 2005; 77:1836-43.

7. Selley ML, Close DR, Stern SE. The effect of increased concentrations of homocysteine on the concentration of (E)-4-hydroxy2-nonenal in the plasma and cerebrospinal fluid of patients with Alzheimer's disease. Neurobiol Aging 2002;23:383-8.

8. Finkelstein JD. Metabolic regulatory properties of S-adenosylmethionine and S-adenosylhomocysteine. Clin Chem Lab Med 2007;45:1694-9.

9. Obeid R, Kostopoulos P, Knapp JP, Kasoha M, Becker G, Fassbender K, et al. Biomarkers of folate and vitamin B12 are related in blood and cerebrospinal fluid. Clin Chem 2007;53: 326-33.

10. Bottiglieri T, Godfrey P, Flynn T, Carney MW, Toone BK, Reynolds EH. Cerebrospinal fluid S-adenosylmethionine in 
depression and dementia: effects of treatment with parenteral and oral S-adenosylmethionine. J Neurol Neurosurg Psychiatry 1990;53:1096-8.

11. Popp J, Lewczuk P, Linnebank M, Cvetanovska G, Smulders Y, Kolsch H, et al. Homocysteine Metabolism and Cerebrospinal Fluid Markers for Alzheimer's Disease. J Alzheimers Dis 2009;18:819-28.

12. Selhub J. Folate, vitamin B12 and vitamin B6 and one carbon metabolism. J Nutr Health Aging 2002;6:39-42.

13. Serot JM, Barbé F, Arning E, Bottiglieri T, Franck P, Montagne $\mathrm{P}$, et al. Homocysteine and methylmalonic acid concentrations in cerebrospinal fluid: relation with age and Alzheimer's disease. J Neurol Neurosurg Psychiatry 2005;76:1585-7.

14. Brattstrom L, Lindgren A, Israelsson B, Andersson A, Hultberg B. Homocysteine and cysteine: determinants of plasma levels in middle-aged and elderly subjects. J Intern Med 1994;236: 633-41.

15. Surtees R, Leonard J, Austin S. Association of demyelination with deficiency of cerebrospinal-fluid S-adenosylmethionine in inborn errors of methyl-transfer pathway. Lancet 1991;338: $1550-4$.

16. Linhart HG, Troen A, Bell GW, Cantu E, Chao WH, Moran E, et al. Folate deficiency induces genomic uracil misincorporation and hypomethylation but does not increase DNA point mutations. Gastroenterology 2009;136:227-35.

17. Serot JM, Christmann D, Dubost T, Bene MC, Faure GC. CSFfolate levels are decreased in late-onset AD patients. J Neural Transm 2001;108:93-9.

18. Hagnelius NO, Wahlund LO, Nilsson TK. CSF/serum folate gradient: physiology and determinants with special reference to dementia. Dement Geriatr Cogn Disord 2008;25:516-23.

19. Taylor MJ, Carney SM, Goodwin GM, Geddes JR. Folate for depressive disorders: systematic review and meta-analysis of randomized controlled trials. J Psychopharmacol 2004;18: 251-6.

20. Smach MA, Jacob N, Golmard JL, Charfeddine B, Lammouchi $\mathrm{T}$, Ben OL, et al. Folate and homocysteine in the cerebrospinal fluid of patients with Alzheimer's disease or dementia: a case control study. Eur Neurol 2011;65:270-8.

21. Ramaekers VT, Blau N. Cerebral folate deficiency. Dev Med Child Neurol 2004;46:843-51.
22. Folstein MF, Robins LN, Helzer JE. The mini-mental state examination. Arch Gen Psychiatry 1983;40:812.

23. Struys EA, Jansen EE, de Meer K, Jakobs C. Determination of S-adenosylmethionine and S-adenosylhomocysteine in plasma and cerebrospinal fluid by stable-isotope dilution tandem mass spectrometry. Clin Chem 2000;46:1650-6.

24. Smith DE, Kok RM, Teerlink T, Jakobs C, Smulders YM. Quantitative determination of erythrocyte folate vitamer distribution by liquid chromatography-tandem mass spectrometry. Clin Chem Lab Med 2006;44:450-9.

25. Ubbink JB, Hayward Vermaak WJ, Bissbort S. Rapid highperformance liquid chromatographic assay for total homocysteine levels in human serum. J Chromatogr 1991;565: 441-6.

26. Smulders YM, Smith DE, Kok RM, Teerlink T, Gellekink H, Vaes $\mathrm{WH}$, et al. Red blood cell folate vitamer distribution in healthy subjects is determined by the methylenetetrahydrofolate reductase $\mathrm{C} 677 \mathrm{~T}$ polymorphism and by the total folate status. J Nutr Biochem 2007;18:693-9.

27. Fazili Z, Pfeiffer CM, Zhang M. Comparison of serum folate species analyzed by LC-MS/MS with total folate measured by microbiologic assay and Bio-Rad radioassay. Clin Chem 2007;53:781-4.

28. Weir DG, Molloy AM, Keating JN, Young PB, Kennedy S, Kennedy DG, et al. Correlation of the ratio of S-adenosyl-Lmethionine to S-adenosyl-L-homocysteine in the brain and cerebrospinal fluid of the pig: implications for the determination of this methylation ratio in human brain. Clin Sci (Lond) 1992;82:93-7.

29. Morrison LD, Smith DD, Kish SJ. Brain S-adenosylmethionine levels are severely decreased in Alzheimer's disease. J Neurochem 1996;67:1328-31.

30. Wollack JB, Makori B, Ahlawat S, Koneru R, Picinich SC, Smith $A$, et al. Characterization of folate uptake by choroid plexus epithelial cells in a rat primary culture model. J Neurochem 2008;104:1494-503.

31. Steinfeld R, Grapp M, Kraetzner R, Dreha-Kulaczewski S, Helms G, Dechent P, et al. Folate receptor alpha defect causes cerebral folate transport deficiency: a treatable neurodegenerative disorder associated with disturbed myelin metabolism. Am J Hum Genet 2009;85:354-63. 